\title{
An Efficient Energy Saving Approach for Improving Lifetime of Node in Wireless Sensor Network
}

\author{
Miss. Diksha R. Nakhale \\ Department of Computer Engineering B.D.C.E. \\ Wardha
}

\author{
Prof. A. N. Thakre \\ Department of Computer Engineering B.D.C.E. \\ Wardha
}

\begin{abstract}
Sleep/wake-up scheduling is one of the fundamental problems in wireless sensor networks, since the energy of sensor nodes is limited and they are usually unrechargeable. The purpose of proposed work is to save the energy of each node by keeping nodes in sleep mode as long as possible (without sacrificing packet delivery efficiency) and thereby maximizing their lifetime. In proposed system, a selfadaptive sleep/wake-up scheduling approach is proposed. Unlike most existing studies that use the duty cycling technique, which incurs a tradeoff between packet delivery delay and energy saving, the proposed approach, which does not us duty cycling, avoids such a tradeoff. In the proposed approach each node autonomously decides its own operation mode like sleep, listen, or transmission in each time slot in a decentralized manner. Simulation results demonstrate the good performance of the proposed approach in various circumstances.
\end{abstract}

Keywords: Energy Efficient, Sleep Awake, Decentralized.

\section{INTRODUCTION}

Due to recent technological advances, the manufacturing of small, low power, low cost and highly integrated sensors has become technically and economically feasible. These sensors are generally equipped with sensing, data processing and communication components. Such sensors can be used to measure conditions in the environment surrounding them and then transform these measurements into signals. The signals can be processed further to reveal properties about objects located in the vicinity of the sensors. The sensors then send these data, usually via a radio transmitter, to a command center also known as a "sink" or a "base station" either directly or via several relaying sensors.

Typically, WSNs contain hundreds or thousands of sensors which have the ability to communicate with each other. The energy of each sensor is limited and they are usually unrechargeable, so energy consumption of each sensor has to be minimized to prolong the life time of WSNs. Major Source of energy waste is idle listening. When a sensor is awake, it is in an idle listening state and it can receive and transmit packets. However, if no packets are received or transmitted during the idle listening time, the energy used during the idle listening time is wasted such waste should certainly be minimized in WSNs, both energy saving and packet delivery delay are important. Because each node in WSNs is usually equipped with an un-rechargeable battery, energy saving is crucial for prolonging the lifetime of WSNs. Because delay is unacceptable in some applications of WSNs, e.g., fire detection and tsunami

Alarm, reducing packet delivery delay is crucial for the effectiveness of WSNs. In proposed system, a self-adaptive sleep/wake-up scheduling approach is proposed, which takes both energy saving and packet delivery delay into account.

This approach does not use the technique of duty cycling most existing studies use the technique of duty cycling to periodically alternate between awake and sleeping states. In most existing duty cycling-based sleep/wake-up scheduling approaches, in duty cycle the time axis is divided into periods, each of which consists of several time slots. In each period, nodes adjust their sleep and wake up time, i.e., adjusting the duty cycle, where each node keeps awake in some time slots while sleeps in other time slots. This approach is first one which does not use duty cycling Instead, it divides the time axis into a number of time slots and lets each node autonomously decide to sleep, listen or transmit in a time slot. Each node makes a decision based on its current situation and an approximation of its neighbors' situations, where such approximation does not need communication with neighbors.

\section{RELATED WORK}

1.Y. Xiao et al., investigates the fundamental performance limits of medium access control (MAC) protocols for particular multihop, RF-based wireless sensor networks and underwater sensor networks. A key aspect of this study is the modeling of a fair-access criterion that requires sensors to have an equal rate of underwater frame delivery to the base station. Tight upper bounds on network utilization and tight lower bounds on the minimum time between samples are derived for fixed linear and grid topologies. The significance of these bounds is two-fold: First, they hold for any MAC protocol under both singlechannel and half-duplex radios; second, they are provably tight. For underwater sensor networks, under certain conditions, author derive a tight upper bound on network utilization and demonstrate a significant fact that the utilization in networks with propagation delay is larger than that in networks with no propagation delay. The challenge of this work about underwater sensor networks 
lies in the fact that the propagation delay impact on underwater sensor networks is difficult to model.

2.S. Zhu, C. Chen, W. Li, B. Yang, and X. Guan concerned with the problem of filter design for target tracking over sensor networks. Different from most existing works on sensor networks, we consider the heterogeneous sensor networks with two types of sensors different on processing abilities (denoted as type-I and type-II sensors, respectively). However, questions of how to deal with the heterogeneity of sensors and how to design a filter for target tracking over such kind of networks remain largely unexplored. We propose in this paper a novel distributed consensus filter to solve the target tracking problem. Two criteria, namely, unbiasedness and optimality, are imposed for the filter design. The so-called sequential design scheme is then presented to tackle the heterogeneity of sensors. The minimum principle of Pontryagin is adopted for type-I sensors to optimize the estimation errors. As for type-II sensors, the Lagrange multiplier method coupled with the generalized inverse of matrices is then used for filter optimization. Furthermore, it is proven that convergence property is guaranteed for the proposed consensus filter in the presence of process and measurement noise. Simulation results have validated the performance of the proposed filter. It is also demonstrated that the heterogeneous sensor networks with the proposed filter outperform the homogenous counterparts in light of reduction in the network cost, with slight degradation of estimation performance.

3.G. Acampora, D. J. Cook, P. Rashidi, and A. V. Vasilakos examine the infrastructure and technology required for achieving the vision of AmI, such as smart environments and wearable medical devices. We will summarize the state-of-the-art artificial intelligence (AI) methodologies used for developing AmI system in the healthcare domain, including various learning techniques (for learning from user interaction), reasoning techniques (for reasoning about users' goals and intensions), and planning techniques (for planning activities and interactions). Author will also discuss how AmI technology might support people affected by various physical or mental disabilities or chronic disease. Finally, we will point to some of the successful case studies in the area and we will look at the current and future challenges to draw upon the possible future research paths.

4. Y. Yao, Q. Cao, and A. V. Vasilakos work in this paper stems from our insight that recent research efforts on open vehicle routing (OVR) problems, an active area in operations research, are based on similar assumptions and constraints compared to sensor networks. Therefore, it may be feasible that we could adapt these techniques in such a way that they will provide valuable solutions to certain tricky problems in the wireless sensor network (WSN) domain. To demonstrate that this approach is feasible, we develop one data collection protocol called EDAL, which stands for Energy-efficient Delay-aware Lifetimebalancing data collection. The algorithm design of EDAL leverages one result from OVR to prove that the problem formulation is inherently NP-hard. Therefore, we proposed both a centralized heuristic to reduce its computational overhead and a distributed heuristic to make the algorithm scalable for large-scale network operations. We also develop EDAL to be closely integrated with compressive sensing, an emerging technique that promises considerable reduction in total traffic cost for collecting sensor readings under loose delay bounds. Finally, we systematically evaluate EDAL to compare its performance to related protocols in both simulations and a hardware testbed.

\section{MOTIVATION}

A wireless sensor network (WSN) consists of spatially distributed autonomous sensors to monitor physical or environmental conditions, such as temperature, sound, pressure, etc. and to cooperatively pass their data through the network to a main location. The more modern networks are bi-directional, also enabling control of sensor activity. The development of wireless sensor networks was motivated by military applications such as battlefield surveillance; today such networks are used in many industrial and consumer applications, such as industrial process monitoring and control, machine health monitoring, and so on.

The WSN is built of "nodes" - from a few to several hundreds or even thousands, where each node is connected to one (or sometimes several) sensors. Each such sensor network node has typically several parts: a radio transceiver with an internal antenna or connection to an external antenna, a microcontroller, an electronic circuit for interfacing with the sensors and an energy source, usually a battery or an embedded form of energy harvesting. A sensor node might vary in size from that of a shoebox down to the size of a grain of dust, although functioning "motes" of genuine microscopic dimensions have yet to be created. The cost of sensor nodes is similarly variable, ranging from a few to hundreds of dollars, depending on the complexity of the individual sensor nodes. Size and cost constraints on sensor nodes result in corresponding constraints on resources such as energy, memory, computational speed and communications bandwidth. The topology of the WSNs can vary from a simple star network to an advanced multi-hop wireless mesh network. The propagation technique between the hops of the network can be routing or flooding.

\section{APPLICATIONS}

\section{Area monitoring}

Area monitoring is a common application of WSNs. In area monitoring, the WSN is deployed over a region where some phenomenon is to be monitored. A military example is the use of sensors detects enemy intrusion; a civilian example is the geo-fencing of gas or oil pipelines.

\section{Environmental/Earth monitoring}

The term Environmental Sensor Networks has evolved to cover many applications of WSNs to earth science research. This includes sensing volcanoes, oceans, glaciers, forests, etc. Some of the major areas are listed below. 


\section{Air quality monitoring}

The degree of pollution in the air has to be measured frequently in order to safeguard people and the environment from any kind of damages due to air pollution. In dangerous surroundings, real time monitoring of harmful gases is an important process because the weather can change rapidly changing key quality parameters.

\section{Characteristics}

The main characteristics of a WSN include:

- Power consumption constrains for nodes using batteries or energy harvesting

- Ability to cope with node failures

- Mobility of nodes

- Communication failures

- Heterogeneity of nodes

- Scalability to large scale of deployment

- Ability to withstand harsh environmental conditions

\section{PROBLEM DEFINITION}

Sleep/wake-up scheduling is one of the fundamental problems in wireless sensor networks, since the energy of sensor nodes is limited and they are usually unrechargeable. The purpose of sleep/wake-up scheduling is to save the energy of each node by keeping nodes in sleep mode as long as possible without sacrificing packet delivery efficiency and thereby maximizing their lifetime. In proposed system, a self-adaptive sleep/wake-up scheduling approach is proposed. Unlike most existing studies that use the duty cycling technique, which incurs a tradeoff between packet delivery delay and energy saving, the proposed approach, which does not us duty cycling, avoids such a tradeoff.

\section{PROPOSED WORK}

In proposed system, a self-adaptive sleep/wake-up scheduling approach is proposed, which takes both energy saving and packet delivery delay into account. This approach is an asynchronous one and it does not use the technique of duty cycling. This approach is the first one which does not use the technique of duty cycling. Thus the tradeoff between energy saving and packet delivery delay, which is incurred by duty cycling, can be avoided. Unlike recent prediction-based approaches, where nodes have to exchange information between each other, this approach enables nodes to approximate their neighbors' situation without requesting information from these neighbors.

\section{Objectives}

To provide energy efficiency for WSN

To implement sleep awake framework for WSN

To implement customize routing based on sleep awake framework
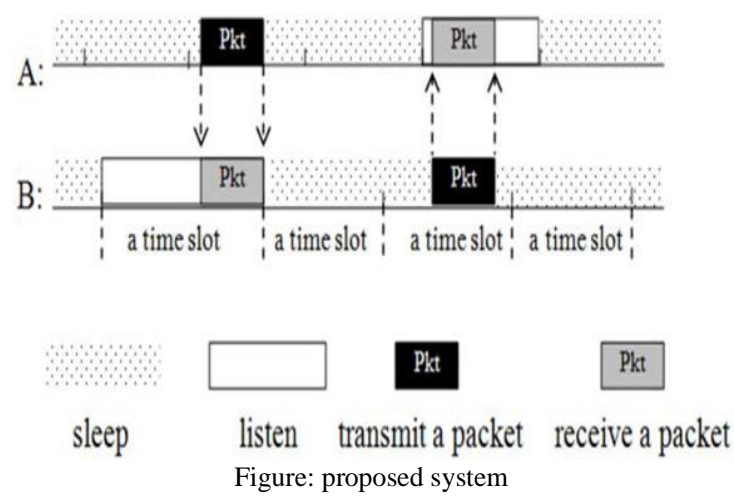

The simulation is operated in grid types of networks: For each type of networks, there are four different scales. The scale of grid networks fluctuatesin 49 nodes, where 49 nodes are structured as a 7 $\times 7$ grid network.In the grid networks, each node is 250 meters from its neighbors and there are 5 sinks which are located at the four corners and the center of the network.Each node generates a packet at the beginning of each time slot based on a predefined probability: the packet generation probability. As the state of a node is determined by the number of packets in its buffer, the packet generation probability directly affects the state of each node. Then, the action selection of each node will be indirectly affected. The expiry time of a packet is based on exponential distribution. The average size of a packet is 100 bytes, and the actual size of a packet is based on normal distribution with variance equal to 10 .

\section{Duty Cycle Scheduling:}

The research of sleep/wake-up scheduling studies how to adjust the ratio between sleeping time and awake time of each sensor in each period.

Sleep: A sensor cannot receive or transmit any packets when it is sleeping, i.e., in sleep state. A sensor in sleep state consumes very little energy.

Transfer/Active: A sensor can receive and transmit packets when it is awake, i.e., in wake-up state. A sensor in wakeup state consumes much more energy compared to sleep state.

Sleep/Wake-Up Scheduling: Sensors adjust the sleeping time length and the awake time length in each period in order to save energy and meanwhile guarantee the efficient transmission of packets.

Generally, the radio transceiver in a sensor node has three modes of operations 1) transmit; 2) listen; and 3) sleep In transmit mode, the radio transceiver can transmit and receive packets. In listen mode, the transmitter circuitry is turned off, so the transceiver can only receive packets. In sleep mode, both receiver and transmitter are turned off. Typically, among these actions, the power required to transmit is the highest, the power required to Figure: Proposed System Flow listen is medium and the power required to sleep is much less compared to the other two actions.

Gossiping: 


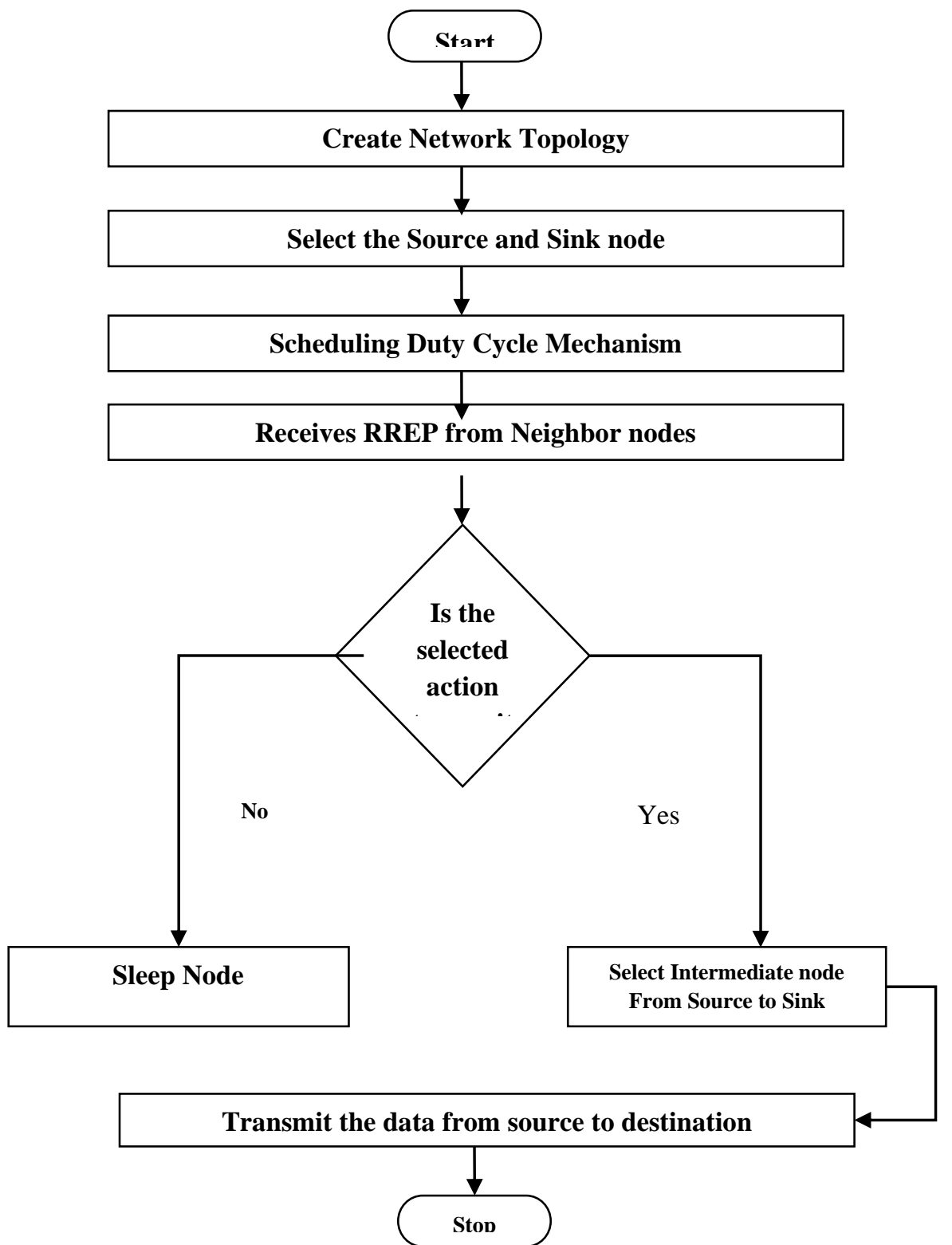

- Average energy consumption. Packet delivery latency is measured by the average time taken by each delivered packet to be transmitted from the source to the destination. Note that those packets, which do not reach the destination successfully, have also been taken into account. Their delivery latency is the time interval, during which they exist in the network. Packet delivery ratio is measured by using the percentage of packets that are successfully delivered from the source to the destination. Average energy consumption is calculated byusingthe total energy consumption to divide the number of nodes in the network during a simulation run

\section{CONCLUSION AND RESULTS}

So by this proposed work energy will be saved, packet dropping rate will be very low and packet delivery delay will be avoided Most existing approaches are based on the duty cycling technique and in this proposed work have taken much effort to improve the performance of their approaches. Thus, duty cycling is a mature and efficient technique for sleep/wakeup scheduling. It is the first one which does not use the duty cycling technique. The performance improvement of the proposed approach, compared with existing approaches in three aspects such as follows

1. Average delivery latency

2. Packet delivery ratio

3 . Average energy consumption
Gossiping is a slightly enhanced version of flooding where the receiving node sends the packet to a randomly selected neighbor, which picks another random neighbor to forward the packet to and so on, until the destination or the maximum hop is reached. It should be noted that when the destination and some other nodes are all in the signal range of the source, based on the outing protocol, the source

still relays a packet to one of neighbors and this process continues until the destination or the maximum hop is reached. The routing process is not optimized in the simulation, as this paper focuses on sleep/wake-up scheduling only. This routing protocol is not energyefficient but it is easy to implement. Because all of the sleep/wake-up scheduling approaches use the same routing protocol in the simulation.

\section{Performance Evaluation}

Performance is measured by three quantitative metrics:

- Average packet delivery latency

- Packet delivery ratio
[1] Y. Xiao et al., "Tight performance bounds of multihop fair access for MAC protocols in wireless sensor networks and underwater sensor networks," IEEE Trans. Mobile Compute., vol. 11, no. 10, pp. 1538-1554,Oct. 2012.

[2] S. Zhu, C. Chen, W. Li, B. Yang, and X. Guan, "Distributed optimal consensus filter for target tracking in heterogeneous sensor networks," IEEE Trans. Cybern., vol. 43, no. 6, pp. 1963 1976, Dec. 2013

[3] G. Acampora, D. J. Cook, P. Rashidi, and A. V. Vasilakos, "A survey on ambient intelligence in healthcare," Proc. IEEE, vol. 101, no. 12, pp. 2470-2494, Dec. 2013

[4] Y. Yao, Q. Cao, and A. V. Vasilakos, "EDAL: An energyefficient, delay-aware, and lifetime-balancing data collection protocol for heterogeneous wireless sensor networks," IEEE/ACM Trans. Netw., vol. 23, no. 3, pp. 810-823, Jun. 2015, doi: 10.1109/TNET.2014.2306592.

[5] S. H. Semnani and O. A. Basir, "Semi-flocking algorithm for motion control of mobile sensors in large-scale surveillance systems," IEEE Trans. Cybern., vol. 45, no. 1, pp. 129-137, Jan. 2015.

[6] B. Fu, Y. Xiao, X. Liang, and C. L. P. Chen, "Bio-inspired group modeling and analysis for intruder detection in mobile sensor/robotic networks," IEEE Trans. Cybern., vol. 45, no. 1, pp. 103-115, Jan. 2015 
[7] Y. Zhao, Y. Liu, Z. Duan, and G. Wen, "Distributed average computation for multiple time-varying signals with output measurements," Int. J. Robust Nonlin. Control, vol. 26, no. 13, pp. 2899-2915, 2016.

[8] Y. Zhao, Z. Duan, G. Wen, and G. Chen, "Distributed finite-time tracking of multiple non-identical second-order nonlinear systems with settling time estimation," Automatica, vol. 64, pp. 86-93, Feb. 2016.

[9] M. Li, Z. Li, and A. V. Vasilakos, "A survey on topology control in wireless sensor networks: Taxonomy, comparative study, andopenissues,"Proc. IEEE, vol. 101, no. 12, pp. 2538-2557, Dec. 2013.

[10] W. Ye, J. Heidemann, and D. Estrin, "An energy-efficient MAC protocol for wireless sensor networks," in Proc. IEEE INFOCOM, New York, NY, USA, Jun. 2002, pp. 1567-1576.

[11] W. Ye, F. Silva, and J. Heidemann, "Ultra-low duty cycle MAC with scheduled channel polling," in Proc. ACM SenSys, Boulder, CO, USA, USA, Nov. 2006, pp. 321-334.

[12] X. Liu, "A deployment strategy for multiple types of requirements in wireless sensor networks," IEEE Trans. Cybern., vol. 45, no. 10 , pp. 2364-2376, Oct. 2015.

[13] C.-P. Chen et al., "A hybrid memetic framework for coverage optimization in wireless sensor networks," IEEE Trans. Cybern., vol. 45, no. 10, pp. 2309-2322, Oct. 2015.

[14] P. Guo, T. Jiang, Q. Zhang, and K. Zhang, "Sleep scheduling for critical event monitoring in wireless sensor networks," IEEE Trans. Parallel Distrib. Syst., vol. 23, no. 2, pp. 345-352, Feb. 2012.

[15] G. Wei, Y. Ling, B. Guo, B. Xiao, and A. V. Vasilakos, "Prediction-based data aggregation in wireless sensor networks: Combining grey model and Kalman filter," Comput. Commun., vol. 34, no. 6, pp. 793-802, 2011.

[16] W. Ye, J. Heidemann, and D. Estrin, "Medium access control with coordinated adaptive sleeping for wireless sensor networks," IEEE/ACM Trans. Netw., vol. 12, no. 3, pp. 493-506, Jun. 2004.

[17] Y. Sun, O. Gurewitz, and D. B. Johnson, "RI-MAC: A receiverinitiated asynchronous duty cycle MAC protocol for dynamic traffic loads in wireless sensor networks," in Proc. ACM SenSys, Raleigh, NC, USA, Nov. 2008, pp. 1-14.

[18] S. Lai, B. Ravindran, and H. Cho, "Heterogenous quorum-based wakeup scheduling in wireless sensor networks," IEEE Trans. Comput., vol. 59, no. 11, pp. 1562-1575, Nov. 2010.

[19] L. Gu and J. A. Stankovic, "Radio-triggered wake-up capability for sensor networks," in Proc. 10th IEEE Real Time Embedded Technol. Appl. Symp., Toronto, ON, Canada, 2004, pp. 27-37.

[20] M. J. Miller and N. H. Vaidya, "A MAC protocol to reduce sensor network energy consumption using a wakeup radio," IEEE Trans. MobileComput., vol. 4, no. 3, pp. 228-242, May/Jun. 2005. 